\title{
Practical utility and reliability of whole-room calorimetry in young children
}

\author{
Xanne Janssen ${ }^{1 *}$, Dylan Cliff ${ }^{1}$, Anthony D. Okely ${ }^{1}$, Rachel A. Jones ${ }^{1}$, Marijka Batterham ${ }^{2}$, Ulf Ekelund ${ }^{3}$, \\ Søren Brage ${ }^{4}$ and John J. Reilly ${ }^{5}$ \\ ${ }^{1}$ Interdisciplinary Educational Research Institute, University of Wollongong, Wollongong, NSW 2500, Australia \\ ${ }^{2}$ Centre for Statistical and Survey Methodology, University of Wollongong, Wollongong, NSW 2500, Australia \\ ${ }^{3}$ Department of Sport Medicine, Norwegian School of Sport Sciences, Ullevål Stadion, Oslo 0806, Norway \\ ${ }^{4}$ MRC Epidemiology Unit, Institute of Metabolic Science, Addenbrooke's Hospital, Cambridge CB2 OQQ, UK \\ ${ }^{5}$ School of Psychology and Health, University of Strathclyde, Jordanhill Campus, Glasgow G13 1PP, UK
}

(Submitted 25 January 2012 - Final revision received 21 May 2012 - Accepted 27 July 2012 - First published online 19 September 2012)

\section{Abstract}

The use of whole-room calorimetry (WRC) in young children can increase our understanding of children's energy balance. However, studies using WRC in young children are rare due to concerns about its feasibility. To assess the feasibility of WRC in young children, forty children, aged 4-6 years, were asked to follow a graded activity protocol while in a WRC. In addition, six children participated in two additional resting protocols to examine the effect of diet-induced thermogenesis on resting energy expenditure (REE) measures and the reliability of REE measurement. Refusals to participate and data loss were quantified as measures of practical utility, and REE measured after an overnight fast and after a 90-min fast were compared. In addition, both were compared to predicted BMR values using the Schofield equation. Our results showed that thirty (78.9\%) participants had acceptable data for all intensities of the activity protocol. The REE values measured after a 90-min fast $(5.07$ (SD 1.04) MJ/d) and an overnight fast (4.73 (SD 0.61) MJ/d) were not significantly different from each other $(P=0.472)$. However, both REE after an overnight fast and a $90-$ min fast were significantly higher than predicted BMR (3.96 (SD 0.18) MJ/d) using the Schofield equation ( $P=0.024$ and 0.042 , respectively). We conclude that, with a developmentally sensitive approach, WRC is feasible and can be standardised adequately even in 4- to 6-year-old children. In addition, the effect of a small standardised breakfast, approximately $90 \mathrm{~min}$ before REE measurements, is likely to be small.

\section{Key words: Activity energy expenditure: Energy metabolism: Pre-school children: Resting energy expenditure}

Whole-room calorimetry (WRC) has been of enormous value in the study of energetics in animal models and in adult human subjects: it has been used to measure energy expenditure, substrate oxidation and energy balance in a wide variety of nutritional applications in many seminal studies $^{(1-3)}$. The use of WRC in children and adolescents has been less common, but, within this realm, has provided important insights into energy metabolism in paediatric obesity $^{(4)}$, and been useful in short-term studies aimed at the validation and calibration of devices to estimate freeliving physical activity and energy expenditure in children and adolescents ${ }^{(5)}$.

Energy balance during the pre-school years (3-5 years) may be a period of the lifecycle which is particularly important to the development of later obesity ${ }^{(6,7)}$; however, the use of WRC in pre-school children at present is limited. There is concern over the feasibility of the use of WRC with young children - the standard fasting requirements might be difficult to adhere to and they may not be able to remain in the WRC for the periods of time that have been used in adult studies. The 'proof of concept' that WRC is feasible in young children has been demonstrated recently ${ }^{(8)}$, but standardised WRC protocols for young children are not available, and some basic issues in the use of WRC in early childhood have not been resolved. For example, it is not clear if it is possible to measure resting energy expenditure (REE) in the calorimeter in young children, given that young children find it difficult to remain still for a required period of time and overnight fasting is not possible. In addition, the compliance with a graded $2 \cdot 5$-h activity protocol - that is, are young children able to reach the required variations in physical activity intensities? - has only previously been examined in a small sample $(n 5)^{(8)}$. The present study, therefore, aimed to establish a protocol for WRC in 4- to 6-year-old children, which would permit the expansion of WRC in this age group in future nutritional research.

Abbreviations: LPA, light physical activity; MVPA, moderate-to-vigorous physical activity; REE, resting energy expenditure; WRC, whole-room calorimetry. 
The study addressed three specific questions: (1) what level of data loss/non-compliance can be expected when conducting WRC studies in a sample of 4- to 6-year-old children?; (2) what is the impact of diet-induced thermogenesis on WRC measurements with a shorter fast (given that it may be impractical to fast young children overnight prior to WRC measures)?; (3) can stable and valid measures of REE be obtained from WRC in this population?

\section{Methods}

\section{Study participants}

The present study was based on a sample of forty healthy 4- to 6-year-old children and was part of a larger study that aimed to validate various objective methods of estimation of freeliving energy expenditure and physical activity in young children. Participants undertook a 150-min protocol (Table 1) in which, following the rest period, they completed activities within the WRC. These were designed to increase their energy expenditure to light $(>1.5$ and $<3$ times predicted BMR) and then to moderate-to-vigorous intensities ( $\geq 3$ times predicted BMR), and to sufficiently sustain these increases in energy expenditure so that at least one of the averaged 10-min samples from the WRC was within the threshold range for each intensity ${ }^{(8)}$.

Table 1. Whole-room calorimetry protocol for the main study*

\begin{tabular}{lc}
\hline & Estimated \\
Activity & time (min) \\
\hline Sedentary intensity & \\
Watching TV - sitting on a beanbag & 30 \\
Talking on telephone with parents - sitting & 2 \\
Reading books with a cassette - sitting & 5 \\
Drawing/colouring in - sitting & 10 \\
Subtotal & 47 \\
Light intensity & \\
Playing with toys, Lego, dolls, puzzles, & 20 \\
$\quad$ games - sitting on floor & 3 \\
Drawing on a whiteboard - standing & 3 \\
Personal grooming (brushing teeth, hair, & \\
$\quad$ washing hands/face) & 5 \\
Dressing up in costumes & 5 \\
Playing musical instruments - standing & 4 \\
Domestic chores (hanging out washing, setting table) & 5 \\
Mini-golf & 5 \\
Walking on spot - light effort (Wii game) & 2 \\
Playing quoits & 5 \\
Subtotal & 5 \\
Moderate and vigorous intensity & 5 \\
Cleaning (packing away toys, dusting, sweeping) & 5 \\
Running on spot - moderate effort (Wii game) & 5 \\
Hopscotch, star jumps, walking stairs & 5 \\
Shooting small basketball into small ring on wall \\
Animal walks (e.g. like a chicken, kangaroo, bear) & 5 \\
Wii sports cycling & 5 \\
Hitting a balloon in the air and catching it & 5 \\
Circuit (walking up foam stairs, jumping off, \\
$\quad$ crawling through a standing hoop, and running back) \\
Running on the spot (Wii game) & 5 \\
Suncing/aerobics (Wii Game) & 5 \\
Grand total & 5 \\
\hline
\end{tabular}

TV, television.

${ }^{*} n$ 40, eighteen girls, twenty-two boys.
Participants were recruited from childcare centres (preschools, long-day and family-day care) in the Illawarra region of New South Wales, Australia (population about $0 \cdot 4$ million). Children were excluded from the study if they had a disease known to influence their energy balance, had a physical disability and/or were claustrophobic. The study was approved by the University of Wollongong/SESIAHS Health and Medical Human Research Ethics Committee, and all participating parents provided informed written consent; their children assented to participation.

\section{Whole-room calorimeter}

$\mathrm{O}_{2}$ consumption and $\mathrm{CO}_{2}$ production were measured continuously (paramagnetic $\mathrm{O}_{2}$ and infrared $\mathrm{CO}_{2}$ analysers; Sable System, Inc.) in a negative-pressure open-system WRC $(3 \mathrm{~m} \times 2.1 \mathrm{~m} \times 2.1 \mathrm{~m})^{(9)}$ and corrected to standard temperature, pressure and dryness. Temperature was maintained at $21^{\circ} \mathrm{C}$ and sampled air was sent through a solid-state gas sample drying system (Peltier dryer; Maastricht Instruments). Flow rates were monitored using Honeywell AWM720 P1 mass flow meters. Flow rate was adjusted to 50 standard litres/min based on pilot trials used to examine the appropriate flow rate for the measurement of $\mathrm{EE}$ in children of this age. The $\mathrm{O}_{2}$ and $\mathrm{CO}_{2}$ analysers were referenced to fresh air for zero readings and against a span gas and $\mathrm{N}_{2}$ every morning prior to data collection. Additional to the ambient air measured for energy expenditure determination, $\mathrm{N}_{2}$ and alpha standard gases were monitored as a continuous check on the precision of calorimeter performance. Alcohol burns were performed periodically, which showed $\mathrm{CO}_{2}$ recovery rates to be within $1 \%$. The chamber air was sampled every $2 \mathrm{~min}$ and rates of $\mathrm{O}_{2}$ consumption and $\mathrm{CO}_{2}$ production were calculated from in- and outflow, as described in the literature ${ }^{(10)}$. The $\mathrm{O}_{2}$ consumption and $\mathrm{CO}_{2}$ production were then averaged over $10 \mathrm{~min}$, and rates of energy expenditure were calculated using the Weir equation ${ }^{(11)}$. The set up of the WRC and the adjustments made to make the facility more child friendly are reported in more detail elsewhere ${ }^{(8)}$.

\section{Study protocol}

Compliance with graded activity protocol. The practical utility assessment (research question 1) was based on data from the children who participated in the main study. Children were asked to fast for $90 \mathrm{~min}$, after which they entered the WRC and followed the 150-min activity protocol (Table 1). Refusals to participate and data loss were quantified as measures of practical utility. The present study estimated data loss/sample attrition over the entire 150-min WRC protocol by expressing the percentage of the sample who provided acceptable energy expenditure data over at least one averaged 10-min period. That is, at rest (sedentary), in light physical activity (LPA) and moderate-to-vigorous physical activity (MVPA) participant data were valid if they had acceptable data for at least one averaged 10-min period in that intensity (all defined on the basis of multiples of predicted BMR from the Schofield equation). 
Overnight fasted compared with 90-min fasted measures. Of the forty children who completed the 150-min WRC protocol, a sub-study was conducted with six children (two girls and four boys). These children were asked to complete two additional 60-min resting protocols to examine the effect of consumption of a light breakfast, about $90 \mathrm{~min}$ before the first measurements of energy expenditure within the WRC (research question 2). The first resting protocol took place after an overnight fast. On the morning of their second visit, parents were asked to give their children a standardised breakfast (approximately $711 \mathrm{~kJ}$ ) at 07.00 hours and only give them sips of water thereafter. On both visits, children and their parents arrived at the WRC at approximately 08.15 hours. Before entering the WRC, children's height and weight were measured using standardised procedures. Height was measured to the nearest $0 \cdot 1 \mathrm{~cm}$ using a portable stadiometer (PE87; Mentone Educational Centre) and weight was measured to the nearest $0 \cdot 1 \mathrm{~kg}$ using a calibrated electronic scale (Tanita BC-418A; Tanita Corporation of America). Children were fitted with an ActiGraph GT3X accelerometer (Actigraph; LLC). The ActiGraph GT3X is an activity monitor, which is attached to an elastic belt and worn on the right hip. It has been shown to be a valid and reliable device to measure children's sedentary behaviour and physical activity levels ${ }^{(12)}$. Children entered the WRC at around 08.30 hours. Children were asked to enter the WRC, sit still on a beanbag and watch a self-selected movie for $60 \mathrm{~min}$, after which they left the room. Children were observed by researchers, one of their parents and filmed during the entire protocol in the WRC.

During the 150-min protocol for the main study, and the 60-min protocol for the sub-study, children were in contact with parents and the researcher via an intercom in an adjoining room. The children could also see their parents at all times through a large window in the adjoining wall ${ }^{(8)}$. In the 150-min protocol for the main study, a trained early childhood educator led the children through the various stages of the protocol.

Measured resting energy expenditure compared with the predicted $B M R$. The third research question of whether or not measures of REE within the WRC were physiologically plausible was examined by comparing them with estimates of BMR predicted from the Schofield equation ${ }^{(13)}$, using both the data from the main study ( $n 40$ potentially available) and from the sub-study ( $n$ 6). Estimation, rather than measurement, of BMR has been the standard practice in most energy expenditure studies in early childhood ${ }^{(14)}$, and is also used widely in studies of older children ${ }^{(15)}$. It has been suggested that the Schofield equation provides unbiased estimates of BMR relative to ventilated hood indirect calorimetry in young children ${ }^{(16)}$. Informal visual inspection of the children being measured has been sufficient to establish that the children are actually at rest in previous studies ${ }^{(17,18)}$, and the method used in the present study was a more formal direct observation using the Children's Physical Activity Rating Scale ${ }^{(19)}$ from the filmed records. The reliability of REE measures within the calorimeter was assessed using repeated 10-min blocks of energy expenditure data from the sub-study.

\section{Data analysis}

Compliance with the resting protocol during measurement of REE was confirmed by visual inspection ${ }^{(4,5)}$. The first 10 min of data within the WRC were seen as the 'settling in'/calorimeter lag period and were not used in the analyses ${ }^{(17,18)}$. In addition, any measurement periods involving large movements were excluded from the data analysis ${ }^{(17)}$. After excluding invalid data defined by direct observation, ActiGraph data were used to verify whether the children were sedentary (counts per $15 \mathrm{~s} \leq 25)$. Mean ActiGraph counts per $15 \mathrm{~s}$ were as follows for the 2nd (95\% CI 10-20 min), 3rd (95\% CI 20-30 min) and 4th (95\% CI 30-40 min) 10-min data blocks of the resting protocols, respectively - overnight fast: 13.3 (95\% CI - 19.3, 45.9), $19 \cdot 8$ (95\% CI - 18.0, 57.6), 6.9 (95\% $\mathrm{CI}-2 \cdot 2,16 \cdot 0)$, and $90-\mathrm{min}$ fast: $5 \cdot 3(95 \% \mathrm{CI}-2 \cdot 4,13 \cdot 1)$, 11.9 (95\% CI $-8 \cdot 5,32 \cdot 4), 14 \cdot 8$ (95\% CI $-5 \cdot 1,34 \cdot 7)$. The results of a repeated-measures ANOVA did not show a significant difference between the 2nd, 3rd and 4th 10-min data blocks of the resting protocol $(P=0.730$ and 0.598 for the overnight fast and 90-min fast REE measures, respectively). With these combined methods, we are confident that the children were resting at the time points that have been included for data analysis.

An average REE was calculated over all the remaining valid data points. A paired-samples $t$ test was used to determine the difference between REE measured after an overnight fast and after a 90-min fast. In addition, paired-samples $t$ tests were used to determine whether there was a difference between estimates of BMR using the Schofield equation and the REE measured within the WRC after an overnight fast and after a 90-min fast. The stability of REE measures within the WRC was tested by calculating intra-individual $\mathrm{CV}^{(17,18)}$. A repeatedmeasures ANOVA was used to determine whether a decline in energy expenditure during the duration of the protocol (as a result of a declining contribution of diet-induced thermogenesis to measured energy expenditure) was discernible.

\section{Results}

\section{Characteristics of participants}

Characteristics of study participants are summarised in Table 2 for both the main study and the sub-study.

\section{Compliance with graded activity protocol}

A total of fifty-four children were identified as being eligible and forty-four agreed to a first familiarisation visit within the WRC, after which forty agreed to participate in the main study. Missing data for the main study was due to calorimeter malfunction ( $n$ 2) or non-compliance. Noncompliance with the activity protocol was a result of not meeting the required intensity; that is, sedentary ( $n$ 5), and/or MVPA ( $n$ 4), of the 150-min protocol, and children ending the protocol early ( $n 2$; Fig. 1 ). This meant that for the thirty-eight children unaffected by calorimeter failures, thirty-three (86.8\%), thirty-six (94.7\%) and thirty-four (89.5\%) complied with the sedentary, LPA and MVPA components of 
Table 2. Subject characteristics

(Mean values and standard deviations)

\begin{tabular}{lrrrrr}
\hline & \multicolumn{2}{c}{$\begin{array}{c}\text { Main study } \\
(n \text { 40) }\end{array}$} & & \multicolumn{2}{c}{$\begin{array}{c}\text { Sub-study } \\
(n 6) \dagger\end{array}$} \\
\cline { 2 - 3 } & Mean & SD & & Mean & SD \\
\hline Age (years) & 5.3 & 1.0 & & 5.5 & 1.1 \\
Height $(\mathrm{cm})$ & 112.7 & 8.1 & & 114.5 & 6.7 \\
Weight $(\mathrm{kg})$ & 20.6 & 3.7 & & 20.6 & 2.5 \\
BMI $\left(\mathrm{kg} / \mathrm{m}^{2}\right)$ & 16.1 & 1.5 & & 15.7 & 1.0 \\
Predicted BMR (MJ/d) & 3.9 & 0.4 & & 4.0 & 0.2 \\
\hline
\end{tabular}

*Eighteen girls, twenty-two boys.

†Two girls, four boys.

the protocol, respectively. The average energy expenditure reached during the protocol was $0.172(\mathrm{SD} 0.038) \mathrm{kJ} / \mathrm{kg}$ per min (1.3 (SD 0.2) predicted BMR), $0.226(\mathrm{SD} 0.029) \mathrm{kJ} / \mathrm{kg}$ per $\min (1.7(\mathrm{SD} 0.2)$ predicted BMR) and $0.402(\mathrm{SD} 0.092) \mathrm{kJ} / \mathrm{kg}$ per min $(3 \cdot 1$ (SD $0 \cdot 8)$ predicted BMR) for sedentary, LPA and MVPA, respectively.

\section{Overnight fasted compared with 90-min fasted measures}

For the sub-study ( $n$ 6), no significant differences were found between REE measured after an overnight fast and after a 90-min fast (4.73 (SD 0.61) and 5.07 (SD 1.04) MJ/d, respectively, $P=0.427$; Fig. 2). There was no significant change in measured REE in both overnight fasted $(P=0.904)$ and 90-min fasted ( $P=0.598)$ states during the duration of the protocol, and therefore there was no indication of a systematic decline in measured REE as a result of a declining contribution of diet-induced thermogenesis (Fig. 3).

\section{Measured resting energy expenditure compared with predicted $B M R$}

The REE measured in the main study, with thirty-three children who fasted for $90 \mathrm{~min}$ prior to the first measurement, was significantly higher than BMR predicted from the Schofield equation (4.73 (SD 1.12) and 3.99 (SD 0.36) MJ/d, respectively, $P<0.001$; Fig. 2). From the sub-study of six children, both the overnight fasted ( 4.73 (SD 0.61$) \mathrm{MJ} / \mathrm{d}$, $P=0.024)$ and $90-\mathrm{min}$ fasted (5.07 (SD 1.04) MJ/d, $P=0.042$ ) measures of REE were significantly higher than predicted BMR (3.96 (sD 0.18) MJ/d; Fig. 2).

\section{Stability of resting metabolic rate measures}

From the sub-sample of six children, the mean intra-individual CV was 14.6 (SD 3.5)\% for REE measured in an overnight fasted state and 9.9 (SD 4.5)\% for REE measured in a 90-min fasted state.

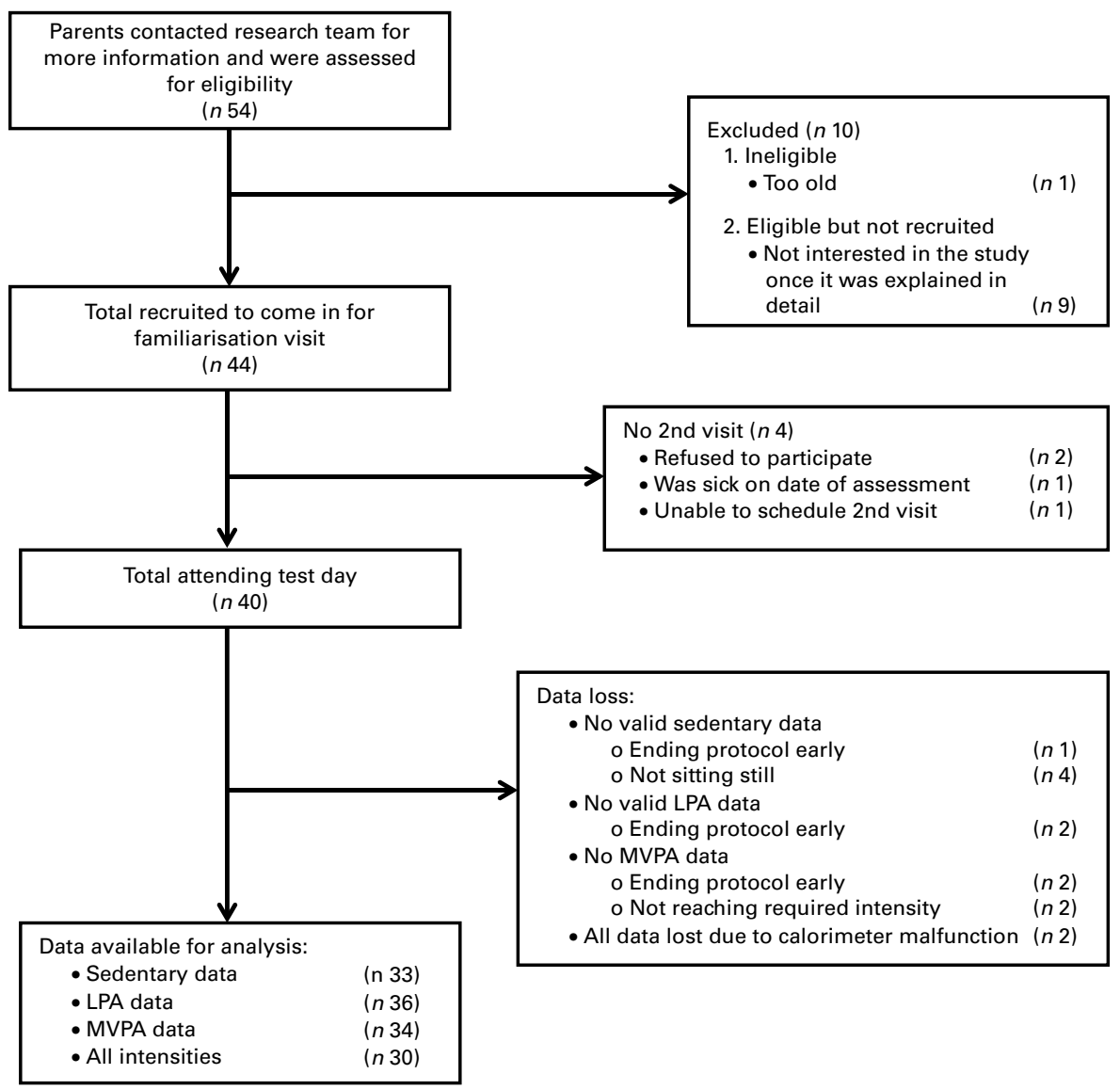

Fig. 1. Flow of participants through the study. LPA, light physical activity; MVPA, moderate-to-vigorous physical activity. 


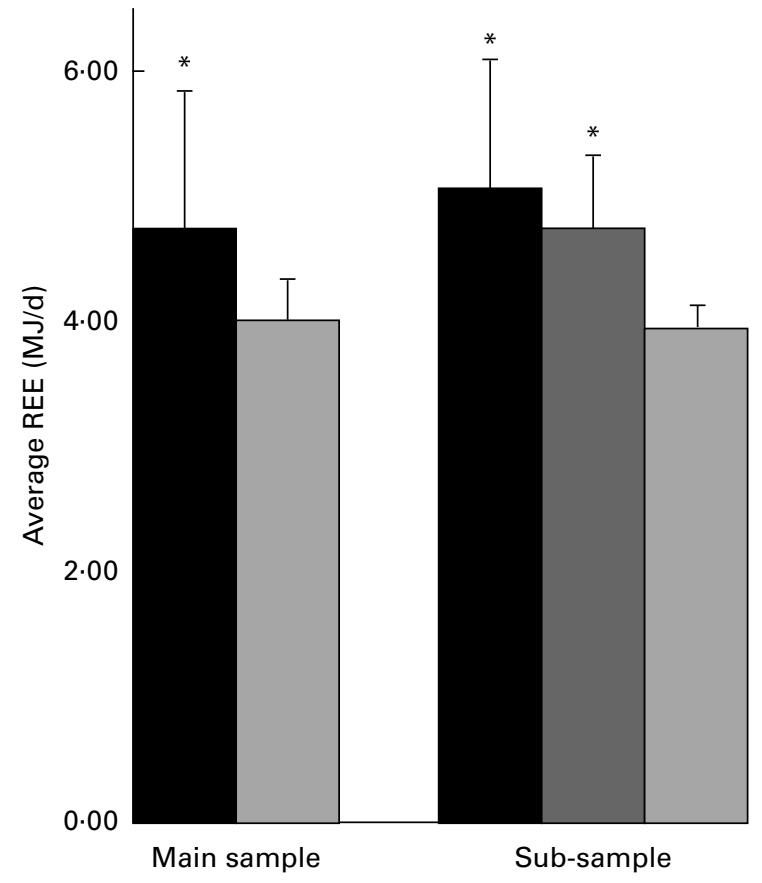

Fig. 2. Mean resting energy expenditure (REE; MJ/d) measured and predicted for both the main sample $(n 33)$ and sub-sample $(n 6)$. ${ }^{*}$ Significantly different from Schofield prediction $(P<0.05)$. . Measured REE after 90-min fast; $\square$, measured REE after overnight fast; $\square$, predicted BMR using Schofield equation.

\section{Discussion}

Our results show that the majority of young children were able to follow the 150-min WRC protocol, which involved a graded transition from resting and sedentary activities to LPA and MVPA, and the 60-min resting protocol. In addition, there was only minor data loss/non-compliance with both WRC protocols. With a developmentally sensitive approach, the WRC is feasible and can be standardised adequately even in 4- to 6-year-old children. These findings confirm and expand on those from a previous pilot study, suggesting that WRC is an option for measurement of energy expenditure in pre-school children ${ }^{(8)}$.

The REE measured in an overnight fasting state was slightly smaller than REE measured after a 90-min fasting state. However, this difference was not statistically significant. This may be attributed to measures beginning at least $90 \mathrm{~min}$ after a light standardised breakfast was consumed, and so dietinduced thermogenesis may have been negligible by the time energy expenditure measures were being conducted. Alternatively, the measurement of energy expenditure may not have been sensitive enough to detect a small decline in REE while fasting (as a result of declining diet-induced thermogenesis), especially with only a small sample. In addition, activity energy expenditure in children is relatively small due to their low body mass and, therefore, even though the difference was not significant, the slightly smaller REE measured after an overnight fast might be of physiological importance. The BMR predicted by the Schofield equation was significantly lower than both REE measured in the overnight fasted $(\Delta 0.77 \mathrm{MJ} / \mathrm{d})$ and 90 -min fasted states
( $\Delta 1.10$ and $0.74 \mathrm{MJ} / \mathrm{d}$ in the sub-sample ( $n$ 6) and main sample ( $n$ 33)), possibly because children were seated rather than lying down. The present study suggests that any effects of diet-induced thermogenesis in energy expenditure data within the WRC $\geq 90$ min after consumption of the light standard breakfast is likely to be small, or at least not detectable. In addition, the reliability of REE measures appeared better in the 90-min fasted condition compared with the overnight fasted condition, and the former is generally more likely to be acceptable to families and human research ethics committees.

Published studies on the use of WRC in 4- to 6-year-old children are unavailable and so no direct comparisons can be made between the present study and other studies. In a study which used a ventilated hood calorimeter in eleven children of average age $4 \cdot 2$ years $^{(17)}$, the highest reliability in REE measures was obtained when data collected during whole body movements were excluded, and, at best, the CV for repeated REE measures was $7 \%$, only a little lower than that observed in the present study for the 90-min fasted protocol. However, in older children, lower CV values $(<4 \%)$ for REE, measured using the ventilated hood, have been reported $^{(18)}$. The slightly increased $\mathrm{CV}$ seen in the present study, compared with the pre-school study of Jackson et $a l .{ }^{(17)}$, might be due to the longer measurement period used and variance within the calorimeter. The reliability of energy expenditure measurement within the calorimeter will influence the ability to detect differences between groups and changes within individuals, but the extent to which this matters will depend on the circumstances. Results from the present study may be useful to future users of WRC in planning how to, or whether to, use such equipment and procedures in young children.

The present study had a number of limitations. We were unable to apply the long-duration (e.g. $24 \mathrm{~h}$ ) protocols and stringent conditions to WRC, which have been typical of adult studies, but this was inevitable given the age of the

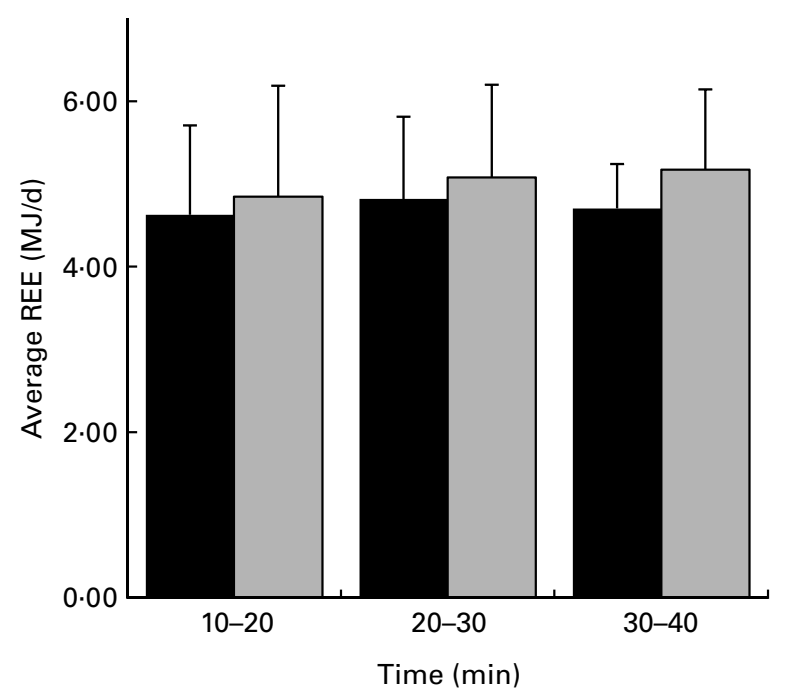

Fig. 3. Resting energy expenditure (REE) at different time points in the fasting protocol ( $n 6)$. $\square$, Overnight fast; $\square, 90$-min fast. 
study participants. Our findings refer specifically to adapted WRC activity protocols of $<3 \mathrm{~h}$ and WRC resting protocols of around $1 \mathrm{~h}$. Generalisability of the present study may be highest when similar samples of young children are studied, and some features specific to the present study (e.g. WRC characteristics) may limit generalisability. The sample of thirty-three children was both relatively large and homogeneous in terms of age compared with previous studies of the reliability of energy expenditure measurement in preschool children $^{(17,18)}$, but it was not possible in the timeframe to recruit more than six children to the sub-study of the effect of diet-induced thermogenesis. With a sample of six, power to detect differences between the two fasting protocols or to detect changes in REE within children over time may have been limited. In addition, REE was not measured while children were lying down: children were reclined on a beanbag while watching a movie. This was considered the most appropriate procedure for this age group, as it would not be feasible for children 4-6 years of age to lie still and recumbent for a prolonged period of time $(30 \mathrm{~min}$, which was needed due to the sample frequency of the calorimeter) without having something (i.e. a movie) to engage their attention. This might have influenced REE measures. The use of the Schofield prediction equation was chosen as a proxy for true or physiological BMR. While one previous study of thirteen pre-school children found no significant difference between Schofield-predicted BMR and ventilatedhood-measured $\operatorname{REE}^{(16)}$, the evidence base which has cross-validated the Schofield equation in this age group is limited. Biases in predictions from the Schofield equation may not be that surprising, and, at the individual level, large errors are to be expected given wide standard errors of the estimate in the Schofield equation.

In conclusion, the use of WRC is feasible and can be standardised adequately even in 4- to 6-year-old children. In addition, the effect of a small standardised breakfast, approximately $90 \mathrm{~min}$ before measurements, is likely to be small. However, this might be of physiological importance and, therefore, we recommend using predicted BMR using the Schofield equation. The outcomes of the present study could help future users in planning how to use WRC in young children.

\section{Acknowledgements}

We would like to thank Harry Battam for his technical support and Melinda Smith for her assistance with recruitment and for leading the participants through the activity protocol. We also thank the children and their parents for their participation. The present study was supported by the National Heart Foundation of Australia (GIA09S4441). D. C. is funded by a National Heart Foundation of Australia - Macquarie Postdoctoral Research Fellowship (PH 09S 4603). A. D. O. is supported by a National Heart Foundation of Australia Career Development Fellowship (CR 11S 6099). The author contributions were as follows: D. C., A. D. O., R. A. J., M. B., U. E., S. B. and J. J. R. designed the research. X. J. conducted the research and analysed the data. X. J. and J. J. R. wrote the manuscript. D. C., A. D. O., R. A. J., M. B., U. E.,
S. B. and J. J. R. reviewed the manuscript and contributed to the discussion. X. J. had primary responsibility for the final content. All authors read and approved the final manuscript. None of the authors had a conflict of interest.

\section{References}

1. Elia M, Stratton R \& Stubbs J (2003) Techniques for the study of energy balance in man. Proc Nutr Soc 62, 529-537.

2. Jebb SA, Prentice AM, Goldberg GR, et al. (1996) Changes in macronutrient balance during over-and underfeeding assessed by 12-d continuous whole-body calorimetry. Am J Clin Nutr 64, 259-266.

3. Stubbs RJ, Hughes DA, Johnstone AM, et al. (2004) A decrease in physical activity affects appetite, energy, and nutrient balance in lean men feeding ad libitum. Am J Clin Nutr 79, 62-69.

4. Butte NF, Puyau MR, Vohra FA, et al. (2007) Body size, body composition, and metabolic profile explain higher energy expenditure in overweight children. J Nutr 137, 2660-2667.

5. Puyau MR, Adolph AL, Vohra FA, et al. (2002) Validation and calibration of physical activity monitors in children. Obes Res 10, 150-157.

6. Reilly JJ (2008) Physical activity, sedentary behaviour and energy balance in the preschool child: opportunities for early obesity prevention. Proc Nutr Soc 67, 317-325.

7. Taylor RW, Goulding A, Lewis-Barned NJ, et al. (2004) Rate of fat gain is faster in girls undergoing early adiposity rebound. Obesity 12, 1228-1230.

8. Oortwijn AW, Plasqui G, Reilly JJ, et al. (2009) Feasibility of an activity protocol for young children in a whole room indirect calorimeter: a proof-of-concept study. J Phys Act Health 6, 633-637.

9. Hill R (1972) Determination of oxygen consumption by use of the paramagnetic analyser. J Appl Physiol 33, 261-263.

10. Schoffelen PFM, Westerterp KR, Saris WHM, et al. (1997) A dual-respiration chamber system with automated calibration. J Appl Physiol 83, 2064.

11. Weir JB (1949) New methods for calculating metabolic rate with special reference to protein metabolism. J Physiol 109, 1-9.

12. Cliff DP, Reilly JJ \& Okely AD (2009) Methodological considerations in using accelerometers to assess habitual physical activity in children aged $0-5$ years. I Sci Med Sport 12, 557-567.

13. Schofield WN (1985) Predicting basal metabolic rate, new standards and review of previous work. Hum Nutr Clin Nutr 39, 5-14.

14. Reilly JJ, Jackson DM, Montgomery C, et al. (2004) Total energy expenditure and physical activity in young Scottish children: mixed longitudinal study. Lancet 363, 211-212.

15. Ball EJ, O'Connor J, Abbott R, et al. (2001) Total energy expenditure, body fatness, and physical activity in children aged 6-9y. Am J Clin Nutr 74, 524-528.

16. Firouzbakhsh S, Mathis RK, Dorchester WL, et al. (1993) Measured resting energy expenditure in children. $J$ Pediatr Gastroenterol Nutr 16, 136-142.

17. Jackson DM, Pace L \& Speakman JR (2007) The measurement of resting metabolic rate in preschool children. Obesity 15, 1930-1932

18. Ventham JC \& Reilly JJ (1999) Reproducibility of resting metabolic rate measurement in children. BrJ Nutr 81, 435-437.

19. DuRant RH, Baranowski T, Puhl J, et al. (1993) Evaluation of the Children's Activity Rating Scale (CARS) in young children. Med Sci Sports Exerc 25, 1415-1421. 